\title{
Enhancing public trust in COVID-19 vaccination during the 2022 FIFA Men's World Cup: a call for action
}

\author{
Mohammad Yasir Essar (D) , ${ }^{1}$ Faisal A. Nawaz, ${ }^{2}$ \\ Salah Eddine Oussama Kacimi, ${ }^{3}$ Selma Nihel Klouche- Djedid, ${ }^{3}$ \\ Jaffer Shah, ${ }^{4}$ Sherief Ghozy, ${ }^{5}$ Edward R. Laskowski ${ }^{6}$
}

The COVID-19 continues to be a global threat with many countries currently battling the third wave of this pandemic. ${ }^{1}$ This pandemic has caused long-term effects in the form of health, human and economic loss along with psychological distress, particularly in low-income countries.

Although vaccination efforts are under way in many countries, vaccine hesitancy, listed as one of global health's most challenging issues, continues to be a major limitation to curbing the pandemic. $^{2}$ Moreover, vaccine distribution inequality has emerged as a matter of serious concern, leaving lower-income countries with limited vaccine doses. ${ }^{3}$

The high visibility of sport provides an opportunity, if not a responsibility, to assist multi-faceted efforts to help mitigate this distressing crisis.

\section{GLOBAL FOCUS: ON FOOTBALL AND HEALTH}

One of the world's most-watched sporting events, the FIFA Men's World Cup, will be hosted in Qatar starting 21 November 2022. The previous edition of this tournament, hosted in Russia, was broadcast to over 3 billion viewers worldwide, ${ }^{4}$ and Qatar is preparing to welcome over 1.2 million visitors for the upcoming competition. $^{5}$

Sporting events have been used in the past as platforms for spreading important

\footnotetext{
${ }^{1}$ Faculty of Dentistry, Kabul University of Medical Sciences, Kabul, Afghanistan

${ }^{2}$ College of Medicine, Mohammed Bin Rashid University of Medicine and Health Sciences, Dubai, United Arab Emirates, Dubai, UAE

${ }^{3}$ Department of Medicine, Faculty of Medicine, University of Tlemcen, Tlemcen (13000), Algeria ${ }^{4}$ New York State Department of Health, Albany, New York, USA

${ }^{5}$ Department of Radiology, Mayo Clinic Minnesota, Rochester, Minnesota, USA

${ }^{6}$ Department of Physical Medicine and Rehabilitation and Division of Sports Medicine, Department of Orthopedics, Mayo Clinic, Rochester, Minnesota, USA
}

Correspondence to Mohammad Yasir Essar, Kabul Medical University, Kabul, Afghanistan; yasir.essar@gmail.com awareness of health issues. This world football championship, involving 32 international teams, is unique given the context of the ongoing pandemic. Currently, FIFA has partnered with the WHO to promote equitable access to vaccines, treatments and diagnostics, and to encourage public health measures to prevent the spread of COVID-19. ${ }^{6}$ This collaboration is further strengthened by FIFA's financial pledge of US \$10 million to support the COVID-19 Solidarity Response Fund. ${ }^{7}$ Thus, the upcoming World Cup presents an exceptional opportunity for COVID-19 health promotion and collaboration with international sports organisations, health authorities, and governments to enhance public vaccine confidence. Vaccines have been shown to remain effective in preventing disease severity, even in the wake of new COVID-19 variants. ${ }^{8}$ Organising a largescale public event like the FIFA Men's World Cup requires comprehensive planning and multidisciplinary efforts to prevent COVID-19, including using the publicity of the event to encourage vaccine uptake. Even though the FIFA World Cup requires the fans and players to be vaccinated for travel, this event still provides an important platform and opportunity to raise awareness on the importance of booster shots, especially given the rising concern for new COVID-19 variants.

\section{POSITIVELY INFLUENCING PUBLIC OPINION}

The role of influencers and activists in the outreach of pro-vaccine messages could act as a powerful education tool for the general public. Following the lead of global health leaders in positively influencing vaccine efforts during the pandemic, a similar strategy can be replicated during global sports events. Although there is a plan to raise awareness on precautions against COVID-19, ${ }^{7}$ half-time intervals during football games can be a crucial window for vaccine-focused promotion using audio and visual announcements. Team players from lower-income countries can further advocate for improved vaccine resources and distribution through this platform to increase awareness in their countries as well as globally. This can help create a ripple effect in combatting vaccine inequity and highlighting the opportunity for support from countries throughout the world. Support from players and staff from higher-income countries can also serve to prompt their countries to develop more strategies to influence vaccine disparity. Similar to social justice issues in which the professional athlete community untied to voice concern and raise awareness, football players at the FIFA Men's World Cup have a similar opportunity to impact the COVID-19 pandemic through the support of important preventive measures. Regulations also are in place to selectively allow only vaccinated attendees, which may serve as a motivating factor for increased vaccination rates within the football community. ${ }^{9}$

\section{PROMOTING SAFETY AND ACCESS}

Integration of global vaccine passports as a means to travel for this event is a controversial measure, and while this qualification may provide an example for other countries and sporting associations to follow, it also must be considered that global inequalities to vaccine access exist and this measure may further widen inequities. Health centres can be established near the stadiums, providing ease and accessibility for public vaccination. Healthcare workers should also be at the forefront of this initiative to harness trust in vaccination. Stalls can be placed by healthcare authorities near stadium entrances for dissemination of brochures and answering public questions related to vaccine safety. Mobile vaccination clinics near the stadium can provide a strategic chance to encourage on-site vaccinations, and an opportunity to vaccinate or provide booster shots to those who do not have ready access to vaccines. Further incentives to motivate football fans in the form of pro-vaccination sporting merchandise can foster a constant source of public interest on this topic. While these on-site vaccination efforts target only a small segment of the population, it demonstrates in real-time and on the world-stage the support of FIFA and other global agencies that vaccines are important.

A recent example of similar health advocacy was observed during the latest edition of the T-Twenty Cricket World Cup. ${ }^{10}$ The use of strategic slogans regarding mental health was strongly beneficial in raising public awareness. A similar theme can be used in the FIFA Men's World 
Cup regarding COVID-19 vaccination and relevant health information. Social media awareness campaigns tailored to football followers with key messages and interviews by players during the tournament could collectively inspire vaccine confidence.

In a world facing a novel pandemic compounded by vaccine hesitancy and misinformation, enhancing public trust in COVID-19 vaccines through innovative measures necessitates a concerted and coordinated call for action. Successful implementation of a large-scale vaccination campaign around a global event will help counter COVID-19 outbreaks, reduce the health burden of this pandemic, and create 'vaccine ambassadors' in the football community to assist in influencing local, regional and even global public policies. It is therefore essential to take advantage of this rare opportunity on a world stage to plan and deliver effective vaccination campaigns to increase public trust.

Correction notice This article has been corrected since it published Online First. Affiliation 4 has been corrected.

Acknowledgements The authors thank the peerreviewers for their constructive feedback that enabled the improvement of the manuscript.

Contributors All authors equally contributed to this work.
Funding The authors have not declared a specific grant for this research from any funding agency in the public, commercial or not-for-profit sectors.

Competing interests None declared.

Patient consent for publication Not applicable.

Ethics approval This study does not involve human participants.

Provenance and peer review Not commissioned; externally peer reviewed.

This article is made freely available for personal use in accordance with BMJ's website terms and conditions for the duration of the covid-19 pandemic or until otherwise determined by BMJ. You may download and print the article for any lawful, non-commercial purpose (including text and data mining) provided that all copyright notices and trade marks are retained.

(c) Author(s) (or their employer(s)) 2022. No commercial re-use. See rights and permissions. Published by BMJ.

$$
\text { D Check for updates }
$$

To cite Essar MY, Nawaz FA, Kacimi SEO, et al. $\mathrm{Br} J$ Sports Med Epub ahead of print: [please include Day Month Year]. doi:10.1136/bjsports-2021-105249

Accepted 11 February 2022

Br J Sports Med 2022;0:1-2.

doi:10.1136/bjsports-2021-105249

ORCID iD

Mohammad Yasir Essar http://orcid.org/0000-00026554-7619

\section{REFERENCES}

1 Naili H. Project HOPE Africa's Third COVID-19 Wave Is Not Over, Continent Should Brace for More Surges, 2021. Available: https://reliefweb.int/report/world/ africa-s-third-covid-19-wave-not-over-continentshould-brace-more-surges [Accessed 06 Nov 2021].

2 Akbar R. WHO ten threats to global health in 2019, 2021. Available: https://www.who.int/news-room/ spotlight/ten-threats-to-global-health-in-2019 [Accessed 06 Nov 2021].

3 Maxmen A. The fight to manufacture COVID vaccines in lower-income countries. Nature 2021:597:455-7.

4 FIFA. More than half the world watched recordbreaking 2018 world cup, 2021. Available: https:// www.fifa.com/tournaments/mens/worldcup/ 2018russia/media-releases/more-than-half-the-worldwatched-record-breaking-2018-world-cup [Accessed 06 Nov 2021].

5 Agence France-Presse. Firstpost FIFA World cup 2022: Qatar hopes to attract 1.2 million visitors for showpiece event, 2021. Available: https://www. firstpost.com/sports/fifa-world-cup-2022-qatar-hopesto-attract-1-2-million-visitors-for-showpiece-event10080461.html [Accessed 6 Nov 2021].

6 FIFA. FIFA and WHO act together to tackle covid-19, 2021. Available: https://www.fifa.com/media-releases/ fifa-and-who-actogether-to-tackle-covid-19 [Accessed 6 Nov 2021].

7 FIFA. Five steps to kicking out coronavirus, 2021. Available: https://www.fifa.com/news/five-steps-tokicking-out-coronavirus [Accessed 6 Nov 2021].

8 Coronavirus Disease 2019(COVID-19) [Internet]. Centers for Disease Control and Prevention, 2021. Available: https://www.cdc.gov/coronavirus/2019ncov/science/science-briefs/scientific-brief-omicronvariant.html [Accessed 28 Dec 2021].

9 Reuters. World cup 2022: Qatar says only fans vaccinated against Covid-19 will be allowed attend matches, 2021. Available: https://www.skysports.com/ football/news/12098/12337288/world-cup-2022qatar-says-only-fans-vaccinated-against-covid-19-willbe-allowed-attend-matches [Accessed 6 Nov 2021].

10 ICC. ICC and UNICEF partner to help break stigma around mental health, 2021. Available: https://www. icc-cricket.com/media-releases/2289193 [Accessed 6 Nov 2021]. 